\title{
Der Widerspruch zwischen nationalen Berufsbildungssystemen und dem europäischen Arbeitsmarkt: der Fall der Maurerqualifikationen
}

Die Berufsausbildungssysteme und die Qualität der Berufsausbildung variieren sehr stark zwischen den Mitgliedsländern der Europäischen Union (EU). Damit stellt sich einerseits die Frage, wie man im europäischen Arbeitsmarkt die unterschiedlichen beruflichen Qualifikationen vergleichen kann. Andererseits wird deutlich, dass die Umsetzung des Europäischen Qualifikationsrahmens (EQR) letztlich auch auf Branchenebene erfolgen muss, was im Folgenden am Beispiel der Bauwirtschaft gezeigt werden soll. ${ }^{1}$

LINDA CLARKE, CHRISTOPHER WINCH, MICHAELA BROCKMANN

\section{Einführung}

Die Freizügigkeit der Arbeitskräfte innerhalb der EU ist untrennbar mit der Notwendigkeit verknüpft, unterschiedliche berufliche Qualifikationen miteinander vergleichen zu können und die Äquivalenz zwischen ihnen zu ermitteln. Ohne eine solche Vergleichbarkeit ist es schwierig für Arbeitgeber, die Fähigkeiten und Kenntnisse ausländischer Stellenbewerber zu beurteilen. Umgekehrt ist es für Arbeitnehmer problematisch, das Niveau ihrer Qualifikationen und deren Stellenwert bzw. Verwendbarkeit in einem anderen Land einzuschätzen. Qualifikationen bilden die Brücke zwischen den Berufsausbildungssystemen und dem Arbeitsmarkt. Und sie reflektieren nicht nur die unterschiedlichen Qualitäten der Arbeitskräfte, sondern auch die Unterschiede zwischen den nationalen Berufsausbildungssystemen. Das eigentliche Problem liegt in den Spannungen zwischen den nationalen Berufsausbildungssystemen und dem europäischen Arbeitsmarkt, da jedes Land trotz der zunehmenden Mobilität der Arbeitskräfte und der zunehmenden Globalisierung der Arbeitsmärkte am eigenen System festhält. Gleichzeitig ist der Arbeitsmarkt auf nationaler und auf europäischer Ebene in unterschiedliche Branchen mit besonderen Interessen und besonderen Organisationsstrukturen der Sozialpartner unterteilt.

Thema dieses Beitrags sind die Spannungen zwischen den nationalen und internationalen Kräften und Interessen speziell im Hinblick auf den Maurerberuf in der Bauwirtschaft. Es soll ermittelt werden, welche Probleme angesichts der nationalen Unterschiede in der Berufsausbildung beim Vergleich der beruflichen Qualifikationen entstehen und wie diese Probleme auf Branchenebene in der EU űberwunden werden können.

Die Analyse und die Empfehlungen, wie eine solche Branchendifferenzierung des EQR aussehen kann, stützen sich auf eine Studie der Autoren, in der sie die Ausbildungsanforderungen für den Maurerberuf in acht Ländern vergleichend untersucht haben (vgl. Clarke et al. 2013). In dieser Studie wurden zunächst die Unterschiede in der institutionellen und konzeptuellen Grundlage der Berufsausbildungs- und Arbeitsmarktsysteme bei der Ausbildung von Maurern auf europäischer, nationaler und betrieblicher Ebene ausgeleuchtet. Hierzu wurden Vertreter der für die Berufsausbildung auf nationaler und europäischer Ebene verantwortlichen Institutionen sowie Vertreter von Ausbildungsanbietern, Arbeitgeberorganisationen und Gewerkschaften befragt. Im zweiten Analyseschritt wurden Fallstudien mit ausführlichen Experteninterviews durchgeführt. Je Land wurden etwa sechs Unternehmen bzw. Baustellen unterschiedlicher Art und Größe, die Maurer beschäftigen, in die Untersuchung einbezogen. Des

1 Übersetzung aus dem Englischen von Angela Grütters. 
Weiteren wurden - gleichfalls je Land - sechs Berufsschulen bzw. Ausbildungseinrichtungen berücksichtigt. In jedem der acht Länder wurden zudem Fokusgruppen eingerichtet, die die jeweiligen nationalen Analyseergebnisse diskutierten und zu deren Interpretation beigetragen haben. In der letzten Phase des Forschungsprozesses wurden die Ergebnisse sowohl innerhalb des internationalen Forschungsteams mehrfach diskutiert als auch mit den Sozialpartnern und Lenkungsgruppen der Baubranche erörtert, um die der jeweiligen Organisation der Arbeitsprozesse zugrunde liegenden Leitideen besser zu verstehen und das Untersuchungsfeld besser einordnen zu können.

\section{Maurerausbildung in der Bauwirt- schaft: „Trades" und Berufe}

Mit rund 14,5 Mio. Beschäftigten ist die europäische Bauwirtschaft ein gutes Beispiel, um zu demonstrieren, wie wichtig und dringlich eine gegenseitige Anerkennung von Qualifikationen geworden ist. Die Arbeitskräfte der Baubranche sind äußerst mobil und wandern im Rahmen ihrer Tätigkeit von Baustelle zu Baustelle und von einem Land zum nächsten. Im Jahr 2007 kam beispielsweise fast eine Viertelmillion entsandter Arbeitskräfte mit einer E101-Bescheinigung ${ }^{2}$ nach Deutschland. Die meisten von ihnen stammten aus Polen, und schätzungsweise mindestens $25 \%$ von ihnen waren im Bausektor tätig. Zur selben Zeit wurde auch in Deutschland eine vergleichbare Zahl von E101-Bescheinigungen ausgestellt (SOKA-BAU 2009). Eine vergleichbare Mobilität herrscht allerorts. Von den ins Ausland entsandten irischen Arbeitnehmern geht die überwiegende Mehrheit ins Vereinigte Königreich, in die Niederlande oder nach Frankreich, wobei knapp die Hälfte der irischen E101-Bescheinigungen für eine Tätigkeit im Bausektor erteilt wird (Cremers 2011). Die Baubranche, die überall eine ähnlich hohe Bedeutung hat und in jedem Land zwischen 5 und $10 \%$ der Erwerbsbevölkerung beschäftigt, ist deswegen eine ideale Branche für den Versuch, Äquivalenz zwischen beruflichen Qualifikationen in Europa herzustellen. Innerhalb dieser Branche ist der Maurerberuf aufgrund seiner universellen Verbreitung das ideale Beispiel.

Um es vorwegzunehmen: Trotz seiner Bedeutung ist das Berufsbild des Maurers in keinem der europäischen Länder identisch. Vielmehr trifft man auf eine Spannbreite von Berufsbildern, die von einfachen Maurertätigkeiten bis hin zu einer breiten Qualifizierung für umfangreiche Bautätigkeiten reichen. Daraus folgt, dass sich auch Berufsausbildung und Qualifikationen für den Maurerberuf stark voneinander unterscheiden. In einigen Ländern, wie beispielsweise in Deutschland, wo 15 \% der Bauarbeitnehmer als Maurer beschäftigt sind, ist es der Bauberuf schlechthin; in anderen Ländern dagegen, wie etwa in den
Niederlanden, wo Zimmerer der wichtigste Bauberuf ist, kommt dem Maurer eine weniger zentrale, aber immer noch wichtige Stellung innerhalb der Branche zu (Brockmann et al. 2010a). Im Extremfall stehen sich zwei völlig unterschiedliche Modelle gegenüber: Auf der einen Seite die Länder, in denen Mauern nach wie vor als traditionelle Tätigkeit gilt, die in der Praxis erlernt wird; auf der anderen Seite gibt es jene Länder, in denen dieser Beruf in berufliche Arbeitsmärkte eingebettet ist mit anerkannten und standardisierten Qualifikationen, einer stabilen Governance-Struktur und der Erwartung, dass das praktische Lernen durch systematisches Wissen fundiert ist (Marsden 2007). In diesem letztgenannten Fall hat sich die Maurertätigkeit zu einem qualifizierten „professionellen“ Beruf entwickelt, der sowohl abstraktes Wissen als auch soziale Kompetenzen voraussetzt.

Zusätzlich zu diesen Unterschieden zwischen den acht Ländern hinsichtlich der heutigen Berufspraxis ist auch eine historisch bedingte Unterscheidung zu erkennen: nämlich zwischen dem Maurerberuf als trade im englischen Sinne (nicht zu verwechseln mit dem deutschen Handwerk) und als qualifiziertem Beruf(Clarke et al. 2013). Von einem als tradesperson geltenden Maurer erwartet der Arbeitgeber, dass er lediglich die Fertigkeiten für typische Maurerarbeiten beherrscht, also Fertigkeiten, die im Rahmen einer traditionellen Lehrlingsausbildung erworben werden können. Dies geschieht hauptsächlich über eine längere Berufspraxis, deren Lernhorizont durch die jeweiligen Arbeitsverfahren begrenzt wird und die ohne nennenswerte theoretische Grundlagen bleibt (Clarke 2005; Brockmann et al. 2010a; Marsden 1999). Ein nach dieser Definition als „tradesperson“ bezeichneter Maurer trägt lediglich die Verantwortung für die ihm zugewiesenen, preislich festgelegten oder vertraglich zugesicherten Aufgaben und muss eine bestimmte Leistung gegen eine entsprechende Vergütung erbringen. Wo die Maurertätigkeit weiterhin auch heute noch als "trade“ definiert wird, ist die Ausbildung dementsprechend relativ eng gefasst und besteht aus induktivem Lernen oder induktiver Generalisierung auf Grundlage von Erfahrungen (Clarke/ Winch 2004). Sie ist häufig auf den spezifischen Bedarf einzelner Unternehmen ausgerichtet, also eher auf eine eng definierte Leistung als auf den Erwerb breit gefächerter beruflicher Fähigkeiten.

Seit die schulische und überbetriebliche Ausbildung integraler Bestandteil einer Berufsausbildung geworden ist, haben sich die Ausbildungssysteme in ganz Europa gewandelt (Steedman 2010). In Deutschland begann diese Entwicklung, unter anderem inspiriert durch Innovationen des damaligen Münchner Stadtschulrats Georg Kerschensteiner, bereits Anfang des 20. Jahrhunderts

2 Die E101-Bescheinigung ist erforderlich, um den jeweiligen Gesundheitsdienst in anderen Ländern in Anspruch nehmen zu können. 
(Hanf 2007; Kerschensteiner 1964), erhielt aber erst mit dem Berufsbildungsgesetz von 1969 einen rechtlichen Rahmen - kurz nach Einrichtung der gesetzlichen „Industrial Training Boards" (Berufsbildungsgremien) im Jahr 1964 in Großbritannien (Greinert 2007; Perry 1976). Zu den Fähigkeiten, die von einem Maurer im Rahmen eines qualifizierten Berufs erwartet werden, gehören die Umsetzung von Wissen in der Praxis sowie die eigenständige Leitung eines Bauprojekts oder von Teilprojekten anstelle eng gesteckter Fertigkeiten, die sich lediglich auf genau definierte Aufgaben beschränken. Von einem qualifizierten Maurer wird also ein umfangreiches berufliches Können erwartet, darunter Tätigkeiten wie Planung, Überwachung, Koordination und Bewertung eines Projekts. All dies erfordert auch die Fähigkeit, Zeichnungen zu verstehen und $\mathrm{zu}$ interpretieren und die Arbeit dementsprechend zu organisieren. Zudem wird Wert auf die Entwicklung individueller Potenziale etwa in Richtung Bauleitung gelegt. Auch wird die Kompetenz erwartet, die laufenden technischen, sozialen und wirtschaftlichen Änderungen zu bewältigen (Brockmann et al. 2008). Dieser Ansatz entspricht auch der Forderung der Gewerkschaften, dass die Berufsausbildung einem Arbeitnehmer umfassende Fähigkeiten vermittelt, sodass er für das gesamte Erwerbsleben, zumindest aber für einen möglichst langen Zeitraum gerüstet ist. Anstatt einer Generalisierung auf Erfahrungsgrundlage basiert ein qualifizierter Beruf meist auf deduktivem Lernen durch Ausprobieren theoretischer Möglichkeiten in praktischen Situationen (Clarke/Winch 2004). Es überrascht indessen nicht, dass der Wissenserwerb größtenteils im Rahmen des Bildungssystems erfolgt, während der Arbeitsprozess und die Werkstättensimulation unerlässlich für die Anwendung des erworbenen Wissens sind.

Trotzdem existiert keine scharfe Trennung zwischen einem trade und einem Beruf. Sie repräsentieren eher zwei Idealtypen, wobei im ersten Fall die Fertigkeiten hauptsächlich im Arbeitsprozess erworben werden, während im zweiten Fall praktische Komponenten nur ein Teil einer umfassenden Berufsausbildung sind. Beide Formen können als Lehrlingsausbildung organisiert werden. Grundlage dafür ist ein Ausbildungsvertrag mit einem Arbeitgeber oder einer Gruppe von Arbeitgebern.

Durch diese Weiterentwicklung von einem traditionellen trade zu einem professionellen Beruf wird von einem Maurer zunehmend eine systematische Berufsausbildung und ein anerkannter Berufsabschluss erwartet. In Deutschland, den Niederlanden und in Skandinavien ist dies auch tatsächlich eine Voraussetzung, um in der Branche eine Beschäftigung finden zu können. Diese Anforderungen haben sich dort durchgesetzt, wo die betriebliche Ausbildung den Hauptweg in den Maurerberuf darstellt, wobei die Ausbildungsgänge zunehmend auf die wechselnden Anforderungen in der sich rasch wandelnden Branche angepasst wurden. Hiermit soll sichergestellt werden, dass die Auszubildenden hinreichend auf die heutzutage geforderten komplexen Aufgaben vorbereitet sind. Von einem Maurer, aber auch von anderen Bauberufen, wird man im Verlauf des nächsten Jahrzehnts unterschiedliche Fähigkeiten verlangen, darunter auch zunehmend autonomes Handeln, um komplexere Anforderungen auf der Baustelle mit hohen Wissensanteilen bewältigen zu können. Beispielsweise erfordert die zunehmende Nutzung von Fertigbauteilen eine präzise Montage sowie Fähigkeiten zum Projektmanagement bei der Montage vor Ort. Im selben Maße erfordert der Einsatz grüner und nachhaltiger Technologien ein umfassenderes Hintergrundwissen, ein Verständnis für den Bauprozess als Ganzes, die reibungslose Kooperation an Schnittstellen mit anderen Tätigkeiten und den Erwerb neuer Spezialkenntnisse (DTI 2008).

\section{Der Maurerberuf und der EQR: drei Typen}

Das wichtigste Instrument der europäischen Politik zur transnationalen Vergleichbarkeit von Qualifikationen ist der 2008 vom Europäischen Parlament verabschiedete und als „Meta-Rahmen“ gestaltete Europäische Qualifikationsrahmen (EQR). Er wurde entwickelt, um innerhalb Europas Qualifikationen vergleichen und akademische und berufliche Qualifikationen in ein gemeinsames Raster einordnen zu können. Dieses Raster umfasst acht Bildungsniveaus mit den Deskriptoren „Kenntnisse“, „Fertigkeiten“ und „Kompetenzen“. Eines der Ziele des EQR ist die Förderung der Arbeitskräftemobilität durch eine höhere Transparenz der Qualifikationsniveaus, wobei der EQR als eine Art „Übersetzungshilfe“ dient (Winch/Mehaut 2011, S. 29). Die EU-Länder können diesen Rahmen freiwillig und nach eigenem Gutdünken als „unterstützenden“ Rahmen verwenden. In der Praxis haben jedoch mittlerweile fast alle Länder nationale Qualifikationsrahmen entwickelt, die zwar mit der Struktur des EQR verflochten sind, aber nicht unbedingt untereinander.

Die transnationale Anerkennung von Qualifikationen im europäischen Arbeitsmarkt auf Ebene von Branchen oder Berufen kann definitionsgemäß nicht anhand eines einfachen Vergleichs von Qualifikationsabschlüssen erfolgen, da diese auf sehr unterschiedlichen nationalen Ausbildungsstrukturen und Standards basieren. Bevor irgendwelche Aussagen zur Äquivalenz bzw. Nichtäquivalenz von Qualifikationen gemacht werden können, muss ein gemeinsamer Analyserahmen erstellt werden, der einen aussagekräftigen Vergleich ermöglicht. Man hofft, dass es Ländern mit sehr ähnlichen Qualifikationen und Berufsausbildungssystemen gelingt, die Probleme des Vergleichens durch sogenannte „Zonen des gegenseitigen Vertrauens“ (,Zones of Mutual Trust“, ZMT) zu überwinden (Coles/Oates 2005). 


\section{Maurerqualifikationen in Europa im Überblick}

\begin{tabular}{|c|c|c|c|c|c|c|c|c|}
\hline Gruppe* & Land & $\begin{array}{c}\text { breit } \\
\text { gefächerter } \\
\text { Lehrplan }\end{array}$ & $\begin{array}{l}\text { Allgemein- } \\
\text { bildung/ } \\
\text { Staatsbürger- } \\
\text { kunde }\end{array}$ & $\begin{array}{c}\text { Projekt- } \\
\text { management }\end{array}$ & $\begin{array}{l}\text { Schulische } \\
\text { Ausbildung }\end{array}$ & $\begin{array}{c}\text { Duale } \\
\text { Ausbildung }\end{array}$ & $\begin{array}{c}\text { Sozialpartner- } \\
\text { schaft }\end{array}$ & $\begin{array}{c}\text { Typisches } \\
\text { Qualifikationsniveau/ } \\
\text { nach dem EOR }\end{array}$ \\
\hline 1 & England & begrenzt & & & vorherrschend & eher selten & $\begin{array}{l}\text { kaum von } \\
\text { Bedeutung }\end{array}$ & 2 gering \\
\hline 1 & Italien & $\begin{array}{c}\text { regionale } \\
\text { Variationen }\end{array}$ & & & & & begrenzt & 2 gering/mittel \\
\hline 2 & Belgien & $\sqrt{ }$ & $\sqrt{ }$ & $\sqrt{ }$ & $\sqrt{ }$ & $\sqrt{ }$ & $\sqrt{ }$ & 3 hoch \\
\hline 2 & Polen & $\sqrt{ }$ & $\sqrt{ }$ & $\sqrt{ }$ & $\sqrt{ }$ & eher selten & begrenzt & 3 hoch \\
\hline $2-3$ & Frankreich & $\sqrt{ }$ & $\sqrt{ }$ & $\sqrt{ }$ & eher selten & $\sqrt{ }$ & $\sqrt{ }$ & 3 hoch \\
\hline 3 & Deutschland & $\sqrt{ }$ & $\sqrt{ }$ & $\sqrt{ }$ & eher selten & $\sqrt{ }$ & $\sqrt{ }$ & 3 hoch \\
\hline 3 & Dänemark & $\sqrt{ }$ & $\sqrt{ }$ & $\sqrt{ }$ & eher selten & $\sqrt{ }$ & $\sqrt{ }$ & $3+$ hoch \\
\hline 3 & Niederlande & begrenzt & $\sqrt{ }$ & $\sqrt{ }$ Niveau 3 & $\sqrt{ }$ & $\sqrt{ }$ & $\sqrt{ }$ & 2 hoch \\
\hline
\end{tabular}

* Diese Spalte bezieht sich auf die Zuordnung der Länder zu den drei generischen Typen. Gruppe 1 steht für Systeme, die auf Beschäftigungsfähigkeit und betrieblicher Ausbildung basieren, Gruppe 2 für schulbasierte Systeme und Gruppe 3 für Länder mit dualem System.

Quelle: Brockmann et al. (2010a).

Derartige Zonen ließen sich auch auf sektoraler Ebene und zwischen unterschiedlichen beruflichen Qualifikationen entwickeln. Eine Studie der Baubranche (vgl. Syben 2009) zeigt, dass für die Umsetzung des EQR auch brancheneigene Qualifikationsrahmen (SQR) erforderlich und vorgesehen sind. Sybens Ergebnisse wie auch unsere eigenen Studien (Clarke et al. 2013) zeigen, dass die Zuordnungen der Qualifikationen im EQR für einzelne Branchen spezifiziert werden müssen. Nur dann wird es möglich sein, die hinter den scheinbar ähnlichen Qualifikationen versteckten Unterschiede im Wissen, den praktischen Fähigkeiten und den individuellen und sozialen Kompetenzen sowie den potenziellen Tätigkeitsfeldern der Ausgebildeten aufzudecken (Brockmann et al. 2010a, S. 21).

Die untersuchten acht Länder lassen sich drei Qualifizierungsmodellen zuordnen, die zu unterschiedlichen Maurerqualifikationen führen. Die Unterschiede beziehen sich auf die im Lehrplan abgedeckten Tätigkeitsbereiche, auf die dem Erwerb von Projektmanagementfähigkeiten beigemessene Bedeutung, auf die Verteilung und Einbeziehung praxis- und unterrichtsbasierter Elemente, auf die Mitwirkung der Sozialpartner sowie auf das Niveau der Qualifikationen und den Arbeitsmarkt (Tabelle 1):

Typ 1: England und teilweise Italien: Die Maurerausbildung hat hier einen geringen Stellenwert, die entsprechende Qualifikation hat ein niedriges Niveau und ist von schmaler Bandbreite; die Mitwirkung der Sozialpartner ist insbesondere in England gering und der Begriff der Kompetenz ist dementsprechend begrenzt (Brockmann et al. 2010b; Verdesca 2010).

Typ 2: Belgien, Frankreich und Polen: Diese Länder sind für ihre schulischen Ausbildungssysteme bekannt. In der Mauerausbildung jedoch ist Frankreich, wie aus Tabelle 1 hervorgeht, atypisch, da hier eine betriebliche Ausbildung mit einem Lehrvertrag der Regelfall ist. ${ }^{3}$ Auch in Belgien ist die Lehrlingsausbildung eine, allerdings untergeordnete Option. Beide Länder stützen sich jedoch auf stabile Institutionen und Praktiken der Sozialpartnerschaft. In allen drei Ländern ist die für das jeweilige Berufsausbildungssystem typische Qualifikation und Kompetenzstruktur breit gefächert und von holistischer Art (Méhaut/Hervy-Guillaume 2010; Janowski/Kus 2010; van Roy 2010).

Typ 3: Dänemark, Deutschland und die Niederlande: Diese Länder sind exemplarisch für eine duale Berufsausbildung. Die Verantwortung wird zwischen Staat und Unternehmen geteilt, man findet eine stark ausgeprägte berufliche Identität und fachliche Breite (wie es sie auch in Frankreich gibt) sowie eine deutliche Vorstellung von beruflicher Kompetenz. Zentrale Strukturmerkmale sind die Sozialpartnerschaft sowie die Integration von theoretischem und praktischem Lernen, wobei in Dänemark geforderte Qualifikationen jedoch von höherem Niveau und breiter gefächert sind (Shapiro et al. 2010; Paul 2010; Westerhuis 2010).

\subsection{Arbeitsmarktbasierte Qualifikationen: England und Italien}

Die englische Berufsausbildung fußt auf schwachen und wenig stabilen Regulierungen und einem - im Vergleich zu anderen Industrieländern - geringen Status der

3 Frankreich würde man, genau wie Polen, normalerweise als typischen Vertreter eines schulbasierten Berufsausbildungssystems betrachten. Im Fall des Maurerberufs überwiegt jedoch auch hier mit ca. $80 \%$ die duale Lehrlingsausbildung. 
beruflichen Ausbildung im Gegensatz zur akademischen Bildung (Brockmann et al. 2009). Bezeichnend für das englische Lehrlingssystem sind geringe Teilnehmerzahlen, mangelndes Engagement seitens der Arbeitgeber, die geringe Bedeutung, die theoretischem Wissen und außerbetrieblicher Bildung beigemessen wird, sowie das allgemein niedrige Qualifikationsniveau, das im Fall des Maurerberufs nur der Stufe 2 und nicht wie in anderen europäischen Ländern der Stufe 3 oder höher des EQR entspricht (Steedman 2010). Die englische Maurerausbildung folgt dem oben beschriebenen Konzept eines „trade“, wobei die Trade-Vereinigungen ${ }^{4}$ des Mauerns, die speziell an Ziegelsteine als Baumaterial gebunden sind, einen maßgeblichen Einfluss auf die Gestaltung der Qualifikationen haben (Brockmann et al. 2010b). Das Trade-Konzept des Maurerberufs ist auch in den Tarifverträgen für die Baubranche erkennbar: Die Tariflöhne, die zwischen den immer weniger repräsentativen Gewerkschaften und Arbeitgeberverbänden ausgehandelt werden, orientieren sich nicht am Qualifikationsniveau. Vergütet wird die konkret geforderte Leistung, d. h. die Erfüllung einer vorgegebenen Aufgabe, sodass der tatsächliche Verdienst möglicherweise kaum den tariflichen Stundenlöhnen entspricht.

Dass Maurer in England meist von reinen Maurerbetrieben oder von Firmen, die nur Arbeiter vermitteln, anstatt von Baufirmen oder Bauauftragnehmern eingestellt werden, verstärkt das Trade-Konzept. Maurer sind zudem oftmals selbstständig und ihre Tätigkeit ist größtenteils auf das Verlegen von Steinen beschränkt. Zudem werden manuelle und intellektuelle Funktionen auf der Baustelle meist getrennt und Tätigkeiten wie Abstecken, Lesen von Zeichnungen, Planung, Kontrolle und Abnahme der Arbeit von Bauleitern, Ingenieuren oder Aufsichtspersonal ausgeführt (Clarke 2005). So entstehen nur wenig direkte Kooperation, Interaktion oder Überschneidung mit anderen Bauberufen. Stattdessen herrscht ein hohes Maß an Spezialisierung vor, die sich in der stark nach Berufen abgegrenzten Ausbildung widerspiegelt. Diese enge Gestaltung der Maurertätigkeit in England findet sich besonders im Wohnungsbau, wo sie sich größtenteils auf das Verlegen von Mauersteinen und -blöcken beschränkt (Clarke/Wall 2000). Im Wirtschaftsbau werden von einem Maurer möglicherweise auch weitere Aufgaben verlangt, wie die Arbeit mit Stein und Beton oder der aufwendigere Bau von Rundbögen.

Die Entwicklung eines stabilen Berufsausbildungssystems wird durch die unregelmäßige Beschäftigung und Gelegenheitsarbeit im englischen Bausektor behindert. Die Maurertätigkeit ist durch ein hohes Maß an informellem Lernen am Arbeitsplatz bestimmt. Viele Maurer haben weder eine Lehre noch irgendeine andere formale Ausbildung absolviert und sind somit auf die Vor-Ort-Beurteilung ihrer Fertigkeiten angewiesen. Dieser Trend ist die logische Folge eines langfristigen Rückgangs der Zahl der Auszubildenden in der Bauwirtschaft und des geringen Stellenwerts einer Maurerausbildung (Gospel 1995).
Wie auch in vielen anderen Ländern (darunter Deutschland und die Niederlande) wird die für die Berufsausbildung und Qualifikationen in der britischen Bauwirtschaft verantwortliche Institution (Sector Skills Council, Construction Skills) über Umlagen und staatliche Fördermittel finanziert, was für England eher ungewöhnlich ist. Diese Institution wird jedoch hauptsächlich von Arbeitgebern mit sehr geringer Beteiligung der Gewerkschaften verwaltet. Die Ausbildung zum Maurer ist auf die Ausführung bestimmter Aufgaben fokussiert und die abschließende Qualifikation (National Vocational Qualification (NVQ), Level 2 Diploma) umfasst zwei schulbasierte Unterrichtsmodule: ein „technisches“ Modul, das minimales Grundwissen lehrt, sowie ein „funktionelles“ Modul, das zu grundlegenden Rechen-, Schreib- und Lesekompetenzen befähigen soll und IKT-Kenntnisse sowie Beschäftigungsfähigkeit („employability“) vermittelt (Kersh/Evans 2010). Allgemeinbildung, Staatsbürgerkunde sowie soziale und persönliche Kompetenzen haben nur geringe Bedeutung (Unwin 2010). Für einen NVQ-Abschluss ist überdies ein Praktikum gefordert. Auszubildende, die keinen Betriebszugang finden, haben später Schwierigkeiten bei der Arbeitsuche. Ein hoher Prozentsatz der Erstausbildung zum Maurer erfolgt an einem College. Rund die Hälfte der Auszubildenden arbeitet im ersten Ausbildungsjahr in einem Betrieb, doch nur ein Teil von ihnen hat einen Lehrvertrag abgeschlossen, während die übrigen auf eine Vor-OrtAkkreditierung der erlernten Fertigkeiten angewiesen sind (Brockmann et al. 2010b, S. 43).

In Italien zeigt der Maurerberuf ebenfalls deutliche trade-Merkmale. Die Organisationsstruktur ist jedoch recht kompliziert, denn sie weist markante regionale Unterschiede auf und ist nach vier nationalen Tarifabkommen für Industrie, Handwerk, KMU (kleine und mittlere Unternehmen) und Arbeitergenossenschaften unterteilt (Verdesca 2010). Wie auch in England spielt der Staat eine minimale Rolle, allerdings wird die Berufsausbildung stärker von den Sozialpartnern durch die Einrichtung von Berufsschulen und mithilfe der Sozialfonds ausgestaltet. In einigen Regionen genießen die Berufsausbildung und die beruflichen Qualifikationen ein höheres Ansehen als in anderen (DTI 2008, S. 29). Die Lehrlingsausbildung, die häufigste Form der Berufsausbildung, erreicht keine hohen Ausbildungszahlen. Inhalt und Struktur werden größtenteils durch regionale Tarifabkommen festgelegt, wobei unterschiedliche Systeme für Jugendliche zwischen 15 und 17 Jahren und für Jugendliche zwischen 18 und 19 Jahren (die sogenannten "professionellen Lehrverträge“) gelten. Auf nationaler Ebene existiert zwar ein „Rahmenabkommen für berufliche Kenntnisse und Kompetenzen“, das jedoch nur wenig oder keine Relevanz für das Berufsaus-

4 Hierzu zählen beispielsweise die Association of Brickwork Contractors, Brick Development Association, Better Brickwork Alliance, Guild of Bricklayers. 
bildungsangebot in den Regionen hat, in denen die Regierung und die Sozialpartner Struktur und Inhalt der Lehrlingsausbildung nach jeweils eigenen Kriterien gestalten. In einigen Regionen, wie beispielsweise in Rom, wo eine starke Sozialpartnerschaft existiert, gibt es jedoch hochwertige Berufsausbildungsmöglichkeiten, die betriebliche und schulische Elemente miteinander kombinieren.

Das italienische Rahmenabkommen unterteilt die Qualifikationen in sieben Kompetenzbereiche (Vorbereitungsschulung, Lesen technischer Zeichnungen, metrische Geometrie für Absteckarbeiten, Werkstoff- und Gerätekunde, Produktionstechnologie, Arbeitsschutz und Kommunikation), die nach Kenntnissen und Fertigkeiten aufgeschlüsselt sind und sowohl berufliches als auch branchenbezogenes Wissen wie bauwirtschaftliche Arbeitsprozesse, beteiligte Akteure und Innovationen in den Produktionsverfahren beinhalten. Ferner sieht dieser Rahmen wichtige technische und generelle Fertigkeiten für Maurer vor, wie den Gebrauch der italienischen Sprache, IKT-Kenntnisse und soziale und persönliche Kompetenzen einschließlich der Fähigkeiten, einen Arbeitsplatz zu suchen, die Arbeitsaufgaben und die eigene Rolle bei der Ausführung zu beurteilen sowie unabhängiges Arbeiten und Teamfähigkeit. Auf mittlerem Qualifikationsniveau umfasst die Maurertätigkeit nach diesem Abkommen die Interpretation von Zeichnungen in den Bereichen Architektur, Zimmerei, Stahlarbeiten, Infrastruktur und Sanierung. Es werden Kompetenzen erwartet für metrische Vermessung von Bauplätzen, Absteckarbeiten, Ausschachtung, Hochbau und Straßenarbeiten, Infrastrukturarbeiten, Montage von Fertigbauteilen u.ä. Der Lehrplan, der deutlich umfangreicher als der in England ist, reflektiert betriebliche und regionale Unterschiede wie die bevorzugte Verwendung bestimmter Baumaterialien. Er ist aber vor allem auch Ausdruck der Stärke und des Einflusses der Sozialpartnerschaft. Dem Tarifabkommen zufolge soll der Maurerberuf für eine Vielfalt von Bautätigkeiten qualifizieren, bei denen unterschiedliche Werkstoffe, unter anderem auch Stein und Marmor, verwendet werden. Wie auch in England ist der Lohn jedoch nicht an Fähigkeiten oder Qualifikationen geknüpft. Stattdessen werden Maurer nach der auszuführenden Arbeit oder Aufgabe bezahlt, obwohl im Tarifabkommen ein Mindestlohn vorgesehen ist. Trotz Beibehaltung starker Trade-Elemente ist das Know-how italienischer Maurer daher komplexer als die trade-basierten Fertigkeiten ihrer englischen Kollegen.

Sowohl das englische als auch das italienische Qualifizierungssystem ist im Wesentlichen output- oder outcome-orientiert. D.h. es ist auf die Ausführung bestimmter Aufgaben ausgerichtet und spiegelt die auf dem Arbeitsmarkt gefragten Fertigkeiten wieder. Der Hauptunterschied zwischen diesen beiden Ländern besteht darin, dass die in England ohnehin schon wenig geschätzte Lehrlingsausbildung weiter an Umfang und Reputation verliert, sodass das collegebasierte System an Bedeutung gewinnt. In Italien verlässt man sich hingegen weiterhin auf die traditionelle Lehrlingsausbildung, deren Kompetenzrahmen - wäre er effektiv - ein wesentlich breiteres Fähigkeitsspektrum und ein breiter gefächertes Grundwissen vermittelt als dies in England der Fall ist. So lassen sich trotz regionaler Unterschiede die italienischen Maurerqualifikationen leichter in einen gemeinsamen, mit dem EQR verknüpften beruflichen Rahmen einordnen als die englischen. Letztere würden aufgrund ihrer unzureichenden Verankerung in sozialpartnerschaftliche Strukturen und ihrer Anfälligkeit für Änderungen wahrscheinlich als minderwertig eingestuft.

\subsection{Auf dualer Ausbildung basierende Quali- fikationen: Dänemark, Deutschland und die Niederlande}

In Dänemark, Deutschland und den Niederlanden basiert die berufliche Ausbildung auf einer stabilen Sozialpartnerschaft und einem gesetzlichen Ordnungsrahmen, der Mindeststandards, Qualifikationsziele und Ausbildungsprogramme festgelegt. Dieser Rahmen ist Ergebnis komplexer, konsensorientierter Vereinbarungen, welche die Anliegen verschiedener Interessengruppen - insbesondere der Gewerkschaften, Arbeitgeber und der Berufsschullehrer - verkörpern. Ihr Anspruch ist, dass die umfassenden, landesweit anerkannten Ausbildungsprogramme eine Brücke zwischen dem Bildungssystem und dem Arbeitsmarkt schlagen. Ein vorrangiges Ziel der Berufsausbildung ist die Erziehung der Auszubildenden zu aktiven Staatsbürgern und ihre Vorbereitung auf den gewählten Beruf. Aus diesem Grund beinhalten die Ausbildungsprogramme der drei Länder soziale, politische und auch allgemeine Bildungselemente zur Förderung der Bildungspotenziale eines jeden Auszubildenden über den Verlauf seines Berufslebens.

Sowohl in Dänemark als auch in Deutschland ist der Beruf des Maurers - im Gegensatz zu England und Italien - eine breit gefächerte Tätigkeit, die innerhalb des allgemeinen Branchenkontexts definiert ist. Ein hervorstechendes Merkmal des deutschen Systems ist das Lernen an unterschiedlichen Orten mit jeweils eigenem Lehrplan, wobei die Lernenden teils in einem Betrieb, teils mit Blockunterricht in überbetrieblichen Ausbildungsstätten und teils in Berufsschulen ausgebildet werden. In Dänemark steht historisch gesehen das schulische Element der Berufsausbildung im Vordergrund, wobei die Auszubildenden zu Beginn mindestens zwanzig Wochen in der Berufsschule verbringen. In beiden Ländern werden breit gefächerte arbeitsplatzbezogene Tätigkeiten mit umfassenden fachspezifischen Kenntnissen kombiniert, die in Berufsschulen unterrichtet werden. Die Berufsausbildung soll einen Maurer dazu befähigen, in Zusammenarbeit mit verwandten Berufen vom Fundament bis hin zum Dach ein Haus zu bauen. Gleichzeitig wird von ihm erwartet, mit verschiedenen Bereichen (Neubau, Renovierung, Restaurierung) und Baumaterialien (wie Ziegel, Stein und Beton) vertraut zu sein. 
Im Gegensatz zum dänischen und deutschen inputbasiertem System liegt der Schwerpunkt in den Niederlanden mehr auf einem ergebnisorientierten Ansatz, d.h. die Qualifikationen definieren sich über detaillierte Tätigkeitslisten und die Anerkennung ist an die erfolgreiche Ausführung der Aufgaben gebunden. Im Gegensatz zu England orientiert man sich jedoch am Leitbild multidimensionaler Kompetenzen. Hierzu wird eine Reihe von Kompetenzen ermittelt, die für den Beruf, das lebenslange Lernen und das Handeln als verantwortungsvoller Bürger erforderlich sind. Vermittelt werden die definierten Kompetenzen durch entsprechende Lehrpläne und selbstbestimmtes Lernen. In den Niederlanden sind Maurerqualifikationen weniger umfassend als in den anderen beiden Ländern, was die stärkere Spezialisierung in der niederländischen Bauindustrie widerspiegelt (Westerhuis 2010).

Am umfassendsten ist wohl die deutsche Qualifikation, die in einem Drei-Stufen-Programm verschiedene Bautätigkeiten abdeckt. Im ersten Jahr werden gemeinsame Grundkenntnisse unterschiedlicher Bauberufe erworben. Im zweiten Jahr spezialisieren sich die Auszubildenden auf einen von drei Bereichen (Hochbau, Tiefbau und Ausbau) und erst im dritten Jahr auf die eigentliche Maurertätigkeit. Die Ausbildungsstätten vermitteln branchenspezifisches Wissen wie Arbeitsrecht und Umweltschutz und decken eine Vielzahl von Tätigkeiten ab. ${ }^{5}$ Die Berufsschule lehrt das theoretische Grundwissen sowie Allgemeinbildung in den Fächern Deutsch, Politik, Wirtschafts- und Sozialwissenschaft, Sport und Religion. In den 1990er Jahren wurden die berufsbezogenen Kenntnisse für eine Maurertätigkeit in 17 Lernfelder unterteilt (z. B. Bau einer Holzkonstruktion, einer einschaligen Wand oder einer geraden Treppe), die auf authentischen Arbeitssituationen basieren und Wissen verschiedener Disziplinen einbeziehen. Dieser handlungsorientierte Lernansatz soll die Integration von Theorie und Praxis erleichtern und die individuelle Kompetenz dank eines umfassenden Verständnisses des gesamten Arbeitsablaufs fördern (Mulder/Sloane 2004).

Im Mittelpunkt der drei Systeme steht ein Kompetenzkonzept, das Wissen, praktisches Know-how sowie persönliche und soziale Fähigkeiten integriert, auch wenn der Begriff der „Kompetenz“ jedes Mal etwas anders definiert wird. In Deutschland ist es die Handlungsfähigkeit, die seit Ende der 1980er Jahre als Leitprinzip des dualen Systems verstanden wird. Mit Handlungsfähigkeit bezeichnet man durchdachtes und sozial verantwortliches Handeln, die Fähigkeit, selbstständige Entscheidungen zu treffen und die Verantwortung für das eigene Tun zu übernehmen sowohl am Arbeitsplatz als auch in der Gesellschaft. Die Berufsausbildung ist daher auf die Entwicklung der Person als Ganzes ausgerichtet, einer Person, die in der Lage ist, uneingeschränkt an der Gesellschaft teilzuhaben, sich dem technologischen und arbeitsorganisatorischen Wandel anzupassen und zunehmend komplexe und unvorhersehbare Situationen in einem sich schnell ändernden Arbeitsumfeld zu bewältigen. Handlungskompetenz wird mittels eines speziellen Ansatzes - unter anderem nach dem Prinzip der „vollständigen Handlung“ - vermittelt, um eine autonome Handlungsweise in Relation zum gesamten Prozess der Planung, Ausführung und Bewertung von Aufgaben zu fördern (Hanf 2009) und so die persönliche Identifizierung mit einem allgemein definierten Berufsfeld (Heinz 1995) mit anerkanntem gesellschaftlichem Status (Greinert 2007) zu erleichtern. Im Gegensatz zu den englischen „skills“ (Fertigkeiten) ist Handlungsfähigkeit daher nicht auf bestimmte Aufgaben bezogen, sondern als Entwicklung des individuellen Potenzials zu verstehen. Das Konzept vereint die getrennten Dimensionen der beruflichen, sozialen und persönlichen Kompetenz, die als übergreifende Prinzipien für den schulischen Teil des dualen Systems dienen. Dort werden sie im Lehrplan und in den Bewertungsmethoden aufgegriffen und auf integrierte Weise weiterentwickelt (Hanf 2009). Der Lehrplan für die betriebliche Ausbildung sieht den Erwerb einer Vielzahl von Fähigkeiten, Kenntnissen und Wissen vor, die in der Praxis erforderlich sind. Die Leistung wird anhand der Lösung exemplarischer Probleme (nicht anhand der Ausführung vorgegebener Aufgaben, wie dies in England der Fall ist) bewertet. Im dualen System sind die Lernenden zwar zugleich Beschäftigte, setzen jedoch ihre Allgemeinbildung fort und werden außerdem in die technischen und betrieblichen Aspekte des Berufs eingeführt.

Kurz gesagt, dieser Typus der Maurerausbildung fußt auf stabilen Strukturen, die von Arbeitgeberverbänden, den Gewerkschaften der Branche und dem Staat, einschließlich der Länder, getragen werden (Hall/Soskice 2001). Innerhalb eines Europäischen Qualifikationsrahmens für die Branche wäre es relativ einfach, eine gegenseitige Anerkennung dieser Maurerqualifikationen $\mathrm{zu}$ erreichen.

\subsection{Bildungsbasierte Qualifikationen: Belgien, Frankreich und Polen}

In Belgien, Frankreich und Polen dominiert im Allgemeinen das schulische System. Dort ist die Berufsausbildung in das allgemeine Bildungssystem integriert, das dem Bildungsministerium unterstellt ist. Die Ausbildung ist breit gefächert und soll junge Menschen zu verantwortungsbewussten Arbeitnehmern und Bürgern erziehen. In Frankreich wird die Maurerqualifikation im Unterschied zur sonstigen Berufsausbildung jedoch zu einem hohen Prozentsatz (80 \%) im Rahmen einer Lehrlingsausbildung erworben. Deswegen ist in Tabelle 1 Frankreich in Gruppe $2-3$ eingeordnet. In Belgien folgen nur rund $40 \%$ der

5 Hierzu zählen die Annahme, Planung und Übergabe der Arbeit, die Einrichtung von Baustellen, Qualitätssicherung Anfertigung und Lesen technischer Zeichnungen, der Bau von Ziegelsteinmauern, Betonieren, Putzarbeiten, Fliesenlegen, Dämmung. 
Kohorte diesem Weg und in Polen ist es lediglich eine kleine Minderheit der angehenden Maurer (Janowski/Kus 2009). In Belgien besteht überdies die Option, ein zusätzliches duales Jahr in einer Berufsschule und einem Betrieb zu absolvieren. In allen drei Ländern haben der betriebliche und der schulische Weg für das Erlernen des Maurerberufs einen gemeinsamen Lehrplan, sodass Lehrlinge von derselben Bildungsbandbreite profitieren. In allen drei Ländern wird die Berufsausbildung bis zu einem gewissen Maße von den Sozialpartnern mitbestimmt. Am deutlichsten ist dies in Belgien, wo die Sozialpartner die Berufsprofile vereinbaren, während die Schulen das Bildungsprogramm gestalten und die Qualifikationsebenen eng an die tarifvertraglichen Lohnstufen gebunden sind (van Roy 2010).

In Belgien und Frankreich gleichen sich die Ausbildungsinhalte. Sie zielen auf die gesamte Person und sollen die Auszubildenden auf ihre Rolle als Beschäftigte und Staatsbürger vorbereiten (Géhin 2007). In Frankreich beinhalten die Curricula sowohl für den schulischen als auch für den betrieblichen Weg zum Maurer daher ein hohes $\mathrm{Maß}$ an Allgemeinbildung (Französisch und eine Fremdsprache, Sport, Geschichte, Geografie und Bürgerkunde) und an theoretischem Wissen (Mathematik und Physik). Bürger- und Sozialkompetenzen sind ebenfalls Teil des Lehrplans und sehen themenspezifische (Information und Kommunikation) sowie allgemeinbildende Lerninhalte vor (Méhaut/Hervy-Guillaume 2010). Von einem französischen „maçon“ (Maurer) wird erwartet, dass er selbstständig arbeiten kann, in der Lage ist, in Kooperation mit verwandten Handwerksberufen ein Haus zu bauen, und unterschiedliche Verfahren beherrscht. ${ }^{6}$ Darüber hinaus sollte ein angehender Maurer Projektmanagementfähigkeiten wie Planung, Controlling, Kommunikation und Interpretation erlernen. Dass er nicht nur zahlreiche verschiedene Arbeitsverfahren beherrschen, sondern auch umfassende Projektmanagementfähigkeiten erlernen muss, lässt darauf schließen, dass die französische Ausbildung dem der dualen Systeme sehr ähnlich ist und sich daher relativ leicht in einen sektoralen europäischen Qualifikationsrahmen einfügen lässt.

Auch in Polen wird erwartet, dass die Ausbildung zum Maurer den jungen Arbeitnehmer nicht nur auf eine Reihe von Arbeitsprozessen, sondern auch auf umfassendes Projektmanagement sowie - mit Fächern wie Arbeitssoziologie und Arbeitspsychologie - auf eine aktive Rolle als Staatsbürger vorbereitet. Die Ausbildung zum Maurer ist in drei Cluster unterteilt: grundlegende Baukenntnisse, Steine verlegen und Verputztechniken sowie wirtschaftliche und professionelle Tätigkeiten. Das erste Cluster - grundlegende Baukenntnisse - umfasst die Grundprinzipien der Bautätigkeit, darunter Werkstoffkenntnisse, Anfertigung und Lesen technischer Zeichnungen und Vermessung. Das zweite Cluster - Steine verlegen und Verputztechniken - beinhaltet ebenfalls berufliche Kenntnisse, bezieht sich jedoch auf spezielle
Tätigkeitsbereiche: ${ }^{7}$ Das letzte Cluster behandelt relevantes Branchenwissen wie grundlegende Wirtschaftskenntnisse, Arbeitsrecht und Arbeitsschutz. Ähnlich wie in Belgien und Frankreich sind auch soziale Kompetenzen wie Kommunikation und Kooperation, Problemlösung, Entscheidungsfindung und ethisches Verhalten im Lehrplan vorgesehen.

In Belgien, Frankreich und Polen ist die Qualifikation breit gefächert, im Bildungssystem verankert und auf die Förderung von Autonomie am Arbeitsplatz und die individuelle Weiterentwicklung des Arbeitnehmers ausgerichtet. Die Unterschiede zwischen diesen Systemen liegen in der relativen Bedeutung der Sozialpartnerschaft und dem Gewicht der Lehrlingsausbildung gegenüber der schulischen Ausbildung. Da die Einstufung im EQR jedoch nicht von den Bildungswegen (den ,inputs“) abhängen soll, kann man ein hohes Maß an formaler Übereinstimmung in den Ausbildungszielen mit den dualen Systemen feststellen. Allerdings hängt der Arbeitsmarkterfolg eines polnischen Maurers auch von seiner Fähigkeit ab, praktische Erfahrungen in der Branche zu sammeln.

\section{Diskussion und Schlussfolgerungen}

Trotz der unterschiedlichen Realitäten, was die Qualifikationen und die Ausbildung zum Maurer wie auch die eigentliche Maurertätigkeit in Europa betrifft, bestehen beträchtliche Ähnlichkeiten zwischen den Ländern. Dies gilt besonders für Belgien, Dänemark, Frankreich, Deutschland und in geringerem Maße auch für die Niederlande und Polen. Die Systeme dieser Länder stützen sich auf die Dualität zwischen Allgemeinbildung und Arbeitsmarkt, zwischen Staat und Wirtschaft bzw. Sozialpartnerschaft und zwischen schulischem, betrieblichem und überbetrieblichem Lernen in Ausbildungsstätten. Hiermit sollen die beruflichen Fähigkeiten und das Potenzial angehender Maurer speziell mit Blick auf die fortlaufende Weiterentwicklung der Branche gefördert und geschützt werden. Allgemeinbildung und persönliche Entwicklung stehen ausdrücklich im Vordergrund der Qualifizierung, die auf einer breit gefächerten und

6 Z.B. die Vorbereitung der Arbeit, Organisation des Arbeits platzes, Entsorgung von Bauabfällen, Gerüstbau, Schalenkonstruktion mit Steinen und Blöcken, Mauerwerk, Armierung, Einschalen, Betonieren, Bauteilmontage, Verputzen und Abdichten, Verlegen von Rohrleitungen, Instandhaltung der Ausrüstung und Informationsaustausch.

7 Z. B. die Organisation und Planung der Arbeit, Baustoffkenntnisse, Erdarbeiten, Steinwände, Decken, Stürze und Gesimse, Holz- und Stahlarbeiten, Betonieren, Putzarbeiten, Ausfugen, Reparatur- und Abbrucharbeiten. 


\section{Eckpunkte für einen sektoralen Qualifikationsrahmen (Bausektor)}

Struktur der Anforderungen

\begin{tabular}{|c|c|c|c|c|}
\hline \multicolumn{3}{|c|}{ Berufliche Kompetenz } & \multicolumn{2}{|c|}{ Persönliche Kompetenz } \\
\hline Wissen/Kenntnisse & Kno & -how & Soziale Kompetenz & Selbstkompetenz \\
\hline $\begin{array}{c}\text { Werkzeuge, } \\
\text { Ausrüstung, } \\
\text { Werkstoffe } \\
\text { Tiefe und Bandbreite, } \\
\text { systematisch nicht } \\
\text { systematisch }\end{array}$ & $\begin{array}{c}\text { Fertigkeiten: } \\
\text { manuell, intellektuell } \\
\text { Spektrum der } \\
\text { auszuführenden } \\
\text { Tätigkeiten }\end{array}$ & $\begin{array}{l}\text { Transversale Fähig- } \\
\text { keiten: } \\
\text { Planung, Organisation, } \\
\text { Controlling, Beurtei- } \\
\text { lung }\end{array}$ & $\begin{array}{l}\text { Team-/Führungs- } \\
\text { qualitäten } \\
\text { Engagement und } \\
\text { Kommunikation }\end{array}$ & $\begin{array}{c}\text { Autonomie/ } \\
\text { Verantwortung, } \\
\text { Erzielen von } \\
\text { Ergebnissen, } \\
\text { Reflexionsvermögen } \\
\text { und Lernkompetenz, } \\
\text { Verantwortlichkeit }\end{array}$ \\
\hline
\end{tabular}

Quelle: Angaben der Autoren.

sich zwangsläufig ändernden Definition der Maurertätigkeit basiert und beträchtliche schulische sowie praktische Elemente (simulierte Tätigkeit in einer Ausbildungswerkstatt) umfasst. Die geforderten Qualifikationen werden von den Sozialpartnern ausgehandelt. Zwar wird die polnische und französische Berufsausbildung stärker vom Staat reguliert, sie ist aber auch auf den Arbeitsmarktbedarf und die Förderung breit gefächerter Fähigkeiten wie berufliches Know-how und soziale und persönliche Kompetenzen ausgerichtet.

Zwischen diesen Ländern ließe sich daher eine „Zone des gegenseitigen Vertrauens“ (ZGV) schaffen, die auf einer vereinbarten Qualifikationsstruktur basiert und, wie in Übersicht 1 gezeigt, nicht nur auf die Maurer, sondern auf die gesamte Branche anwendbar wäre. In diesem Fall würde eine ZGV eine Auswahl nationaler oder sektoraler Berufsausbildungssysteme mehrerer Länder umfassen, die auf Grundlage von Gemeinsamkeiten ihrer beruflichen Qualifikationen und Ausbildungssysteme formale oder informelle Anerkennungsvereinbarungen schließen könnten. Übersicht 1 zeigt einen Vorschlag der Autoren für einen europäischen sektoralen Rahmen für Maurerqualifikationen, der - wenngleich er detaillierter ist - in die EQR-Struktur integriert werden könnte. Dieser Rahmen ermöglicht den sofortigen Vergleich von zwei oder mehr unterschiedlichen nationalen Maurerqualifikationen und könnte als Grundlage für entsprechende Anerkennungsvereinbarungen dienen. Abgesehen von den erforderlichen aufgabenbasierten Tätigkeiten sind auch allgemeine Fähigkeiten, die für den Erwerb fast aller untersuchten Qualifikationen verlangt werden sowie die in englischen Qualifikationen vorherrschenden Fertigkeiten einbezogen.

England, und vielleicht in geringerem Maße auch Italien, würden allerdings außerhalb einer solchen Zone stehen. In diesen beiden Ländern besteht die Gefahr einer Dequalifikation und des Verlustes beruflicher Handlungs- fähigkeit, wenn keine umfassendere Bildungs- und Wissenskomponente zur Bewältigung des Wandels in der Baubranche eingeführt wird (DTI 2008). Die Tendenz in England, die Ausbildung für Bauberufe zunehmend in die Schule zu verlegen, bringt sie der polnischen Ausbildung näher, wobei allerdings deren Bandbreite fehlt. Für die Einordnung in den EQR ist dann möglicherweise zwischen einem Maurer mit niedrigerem Qualifikationsniveau, das dem Konzept des „trade“ entspricht, und einem unabhängigen, berufsorientierten Maurer zu unterscheiden.

Da der EQR auf sektoraler und auf beruflicher Ebene eingesetzt werden soll, muss er Unterschiede im Hinblick auf Tätigkeitsspektrum, Wissen sowie Fähigkeiten im Bereich des Selbst- und Projektmanagements aufzeigen, um einen sinnvollen Vergleich anstellen und Äquivalenz oder Nichtäquivalenz feststellen zu können. 


\section{LITERATUR}

Brockmann, M./Clarke, L./Hanf, G./Méhaut, P./Westerhuis, A./Winch, C. (2011): Knowledge, Skills, Competence in the European Labour Market: What's in a Qualification?, Oxford

Brockmann, M./Clarke L./Winch C. (2008): Knowledge, Skills, Competence: European divergences in vocational education and training - the English, German and Dutch cases, in: Oxford Review of Education 34 (5), S. 547-567 Brockmann M./Clarke L./Winch C. (2009): Competence and competency in the EQF and in competence-based VET systems: different conceptions and realities in England, France, the Netherlands and Germany, in: Journal of European Industrial Training 33 (8/9), S. 787-799

Brockmann, M./Clarke, L./Winch, C. (Hrsg.) (2010a): Bricklaying is more than Flemish bond: bricklaying qualifications in Europe, CLR, Brussels Brockmann, M./Clarke L./Winch C. (2010b): Bricklaying Country Report England, www.bricklayer-Ilp.eu

Clarke, L. (2005): From Craft to Qualified Building Labour in Britain: a comparative approach, in: Labor History 46 (4), S. 473-494

Clarke, L./Wall, C. (2000): Craft versus Industry: the division of labour in European housing construction, in: Construction Management and Economics $18(6)$, S. $689-698$

Clarke, L./Winch, C. (2004): Apprenticeship and Applied Theoretical Knowledge, in: Educational Philosophy and Theory 36 (5), S. 509-521

Clarke, L./Winch, C. (Hrsg.) (2007): Vocational Education: international approaches, developments and systems, Oxford

Clarke L./Winch C./Brockmann M. (2013): Trade-based skills versus Occupational Capacity: the example of bricklaying in Europe, in: Work, Employment and Society (im Erscheinen)

Coles, M./Oates T. (2005): European reference levels for education and training: Promoting credit transfer and mutual trust. Eine von der Qualifications and Curriculum Authority in Auftrag gegebene Studie: Amt für amtliche Veröffentlichungen der Europäischen Gemeinschaften, Luxemburg

Cremers, J. (2011): In search of cheap labour in Europe: working and living conditions of posted workers, CLR Studies (6), Brussels

Danish Technological Institute (DTI) (2008): Future Qualifications and Skills Needs in the Construction Sector, Danish Technological Institute

Géhin, J.-P. (2007): Vocational Education in France: a turbulent history and a peripheral role, in: Clarke, L.Winch, C. (Hrsg.): Vocational Education, a.a.O., S. $34-48$

Gospel, H. (1995): The decline of apprenticeship training in Britain, in: Industrial Relations Journal 26 (1), S. 32-44

Greinert, W.-D. (2007): The German Philosophy of Vocational Education, in: Clarke, L.Winch, C. (Hrsg.): Vocational Education, a.a.O., S. 49-61

Hall, D./Soskice, D. (2001): Varieties of Capitalism: the institutional foundations of comparative advantage, Oxford

Hanf, G. (2007): Under American influence? The making of modern German training in large Berlin enterprises at the beginning of the twentieth century, in: Clarke, L./Winch, C. (Hrsg.): Vocational Education, a.a.O. S. 92-104 Hanf, G. (2009): Cross-national equivalence of vocational skills and qualifications - The case of Germany, unveröffentlichter Bericht: University of Westminster

Heinz, W. R. (1995): Arbeit, Beruf und Lebenslauf - Eine Einführung in die berufliche Sozialisation, Weinheim/München

Janowski, Z./Kus, J. (2010): Bricklaying Country Report Poland, Budowlani, www.bricklayer-Ilp.eu

Kerschensteiner, G. (1964): Ausgewählte Pädagogische Texte, Band 1, Paderborn

Kersh, N./Evans, K. (2010): Facilitating Learners' Motivation and Competence Development in the Workplace: the UK context, in: Rauner, F/Smith, E. (Hrsg): Rediscovering Apprenticeship, Dordrecht, S. 149-160
Marsden, D. (1999): Theory of Employment Systems: microfoundations of societal diversity, Oxford

Marsden, D. (2007): Labour market segmentation in Britain: the decline of occupational labour markets and the spread of ,entry tournaments', in:

Économies et Sociétés 28 (6), S. 965-998

Méhaut, P./Hervy-Guillaume, D. (2010): Bricklaying Country Report France, www.bricklayer-llp.eu

Mulder, R./Sloane, P. (2004): New Approaches to Vocational Education in Europe, Oxford

Paul, V. (2010): Bricklaying Country Report Germany: BIBB,

www.bricklayer-Ilp.eu

Perry, P. J. C. (1976): The Evolution of British Manpower Policy: From the Statute of Artificers to the Industrial Training Act 1964, Portsmouth Shapiro, H./Jensen, F./Buch, S. (2010): Bricklaying Country Report Denmark, www.bricklayer-Ilp.eu

SOKA-BAU (2009): Activity Report, Wiesbaden

Steedman, H. (2010): The State of Apprenticeship in 2010. International Comparison: Centre for Economic Performance, London School of Economics and Political Science

Syben, G. (2009): Sectoral Qualifications Framework for the Construction Industry in Europe: BAQ Forschungsinstitut, Bremen

van Roy, K. (2010): Bricklaying Country Report Belgium, www.bricklayer-Ilp.eu Verdesca, D. (2010): Bricklaying Country Report Italy, www.bricklayer-Ilp.eu Westerhuis, A. (2010): Bricklaying Country Report Netherlands, ECBO, www.bricklayer-Ilp.eu

Winch, C./Méhaut, P. (2011): EU Initiatives in Cross-national Recognition of Skills and Qualifications, in: Brockmann, M./Clarke, L./Hanf, G./Méhaut, P./ Westerhuis, A.Minch, C. (2011): Knowlede, a.a.O., Kapitel 2, S. 22-35 Unwin, L. (2010): Learning and Working from the MSC to New Labour:Young People, Skills and Employment, in: National Institute Economic Review, 212 (1), S. $50-60$

\section{AUTOREN}

LINDA CLARKE, Dr., Professor of European Industrial Relations, Westminster Business School, University of Westminster. Arbeitsschwerpunkte: Berufsbildung, Bauarbeit, Arbeitsgeschichte.

clarkel@wmin.ac.uk

CHRISTOPHER WINCH, Professor of Educational Philosophy and Policy, King's College London. Arbeitsschwerpunkte: Erziehungsphilosophie, berufliches Wissen, Berufsbildungspolitik.

christopher.winch@kcl.ac.uk

MICHAELA BROCKMANN, Dr., Lecturer, Southampton Education School, University of Southampton. Arbeitsschwerpunkte: Erziehungssoziologie, Berufsausbildung, Lernkultur.

m.brockmann@soton.ac.uk 